\title{
Association Between Haemoglobin Variability and Clinical Outcomes in Chronic Kidney Disease
}

\author{
Roshini Malasingam, David W. Johnson and Sunil V. Badve \\ Princess Alexandra Hospital, Brisbane, QLD, \\ Australasian Kidney Trials Network, The University of Queensland, Brisbane, QLD, \\ Australia
}

\section{Introduction}

Anaemia is a common complication of chronic kidney disease (CKD). The underlying physiology related to anaemia in CKD is secondary to reduction in endogenous erythropoietin as the glomerular filtration rate (GFR) declines. The introduction of erythropoiesis stimulating agents (ESA) has revolutionized the management of anaemia in CKD, leading to substantial reductions in the blood transfusion requirements, improvement in energy and physical function and small improvements in health-related quality of life (Clement et. al., 2009; Eschbach et. al., 1987; Gandra et. al., 2010). Targeting higher haemoglobin with ESA therapy has been associated with increased risks of stroke, vascular access thrombosis, hypertension and possibly death (Badve et. al., 2011; Besarab et. al., 1998; Palmer et. al., 2010; Pfeffer et. al., 2009; Phrommintikul et. al., 2007; Singh et. al., 2006). The current KDOQI Clinical Practice Guideline recommends a haemoglobin target of $11-12 \mathrm{~g} / \mathrm{dL}$. However, a substantial proportion of non-dialysis and dialysis CKD patients exhibit fluctuations in the haemoglobin levels, also known as haemoglobin variability. There is an emerging body of evidence demonstrating an association between haemoglobin variability and mortality in CKD patients treated with ESAs. Maintaining haemoglobin levels within narrow target range remains a major challenge in clinical practice. The aim of this chapter is to review the definition, prevalence, risk factors of haemoglobin variability, and its impact on survival, provide recommendations where possible and suggest directions for future research.

\section{Haemoglobin variability}

\subsection{Definition}

The definition of haemoglobin variability is not entirely clear and various studies have used different definitions. Intra-individual haemoglobin variability is defined as the fluctuation of haemoglobin above or below (Kalantar-Zadeh \& Aronoff 2009) or even within the target range over time. Methods to quantify haemoglobin variability are summarised below.

1. Standard deviation of the differences between observed haemoglobin values and haemoglobin slope which represents the mean haemoglobin change over time (Yang et. al., 2007). 
2. Intra-individual standard deviation of 3-month haemoglobin rolling average (Lacson et. al., 2003).

3. Using 3 haemoglobin groups (above/below/within the target) for each of the 6 consecutive monthly haemoglobin values, classification of all patients into 6 groups: (i) consistently low, (ii) consistently high, (iii) consistently within the target range, (iv) low amplitude fluctuation with low haemoglobin values (all-6 mo with low or target-range haemoglobin values), (v) low amplitude fluctuation with high haemoglobin values (all6 mo with high or target-range haemoglobin values), and (vi) high amplitude fluctuation with high haemoglobin values (low, high and target-range haemoglobin values within 6-mo period) (Ebben et. al., 2006).

4. Time-in-target haemoglobin range (De Nicola et. al., 2007).

5. Determining velocity (or deflection) of haemoglobin change by averaging slopes between successive haemoglobin values (Lau et. al., 2010).

6. Change in haemoglobin in 6-mo above and below the reference range (based on the median change in haemoglobin in 6-mo) (Regidor et. al., 2006).

\subsection{Prevalence}

Haemoglobin variability is common not only in patients with end-stage kidney disease (ESKD) on dialysis, but also in CKD patients who are not yet on dialysis (non-dialysis CKD). The reported prevalence of haemoglobin variability in non-dialysis CKD patients varies between 61 to 86\% (Boudville et. al., 2009; Minutolo et. al., 2009). On the other hand, 82 to $90 \%$ of ESKD patients on dialysis exhibit haemoglobin variability (Ebben et al., 2006; Eckardt et. al., 2010; Gilbertson et. al., 2009).

\section{Risk factors}

\subsection{ESA therapy}

Requirement of ESA for the treatment of anaemia in CKD is a major risk factor for haemoglobin variability. In a study involving 6,165 non-dialysis CKD patients, only $47 \%$ of patients who were not treated with any ESAs demonstrated fluctuations in haemoglobin (Boudville et al., 2009). However, $73 \%$ of patients who were already treated with ESAs experienced haemoglobin variability. This prevalence further increased to $77 \%$ among individuals who were commenced on ESAs as a new therapy. Patients treated with ESA therapy for a longer duration were less likely to have haemoglobin variability. Each 3-mo increment in the duration of ESA therapy decreased the risk of haemoglobin variability by $6 \%$. In a study involving 5,037 ESKD patients on haemodialysis, the risk of developing haemoglobin variability was more than twice in patients on ESAs, compared to those not on ESAs (Eckardt et al., 2010). These findings suggest that the need of ESA rather than ESA therapy per se leads to haemoglobin variability (See Table 1).

There is increased interest in studying the effect of various anaemia management protocols on haemoglobin variability. Patel and colleagues studied the effect of route of administration of erythropoietin on haemoglobin variability in a post hoc analysis of a randomised controlled trial involving 157 prevalent ESKD patients on dialysis (Patel et. al., 2009). Over a follow up of 24-weeks, compared to patients treated with intravenous erythropoietin, those treated with subcutaneous erythropoietin were more likely to have (i) 


\begin{tabular}{|l|}
\hline Factors related to ESA therapy \\
Route of administration \\
Long versus short acting ESA \\
Responsiveness to the first dose of ESA \\
Haemoglobin before initiation of ESA \\
Iron supplementation \\
Frequency of the ESA dose adjustments \\
Magnitude of the ESA dose adjustments \\
Patient-level factors \\
Age \\
Gender \\
Body mass index \\
Incident versus prevalent dialysis \\
Comorbid conditions \\
Number and duration of hospitalisation \\
Use of catheter as haemodialysis vascular access \\
Facility-level anaemia management protocols \\
Proportion of patients prescribed ESAs \\
Frequency of haemoglobin monitoring \\
Frequency of ESA dose monitoring \\
Wider range of target haemoglobin \\
Higher upper target range of haemoglobin
\end{tabular}

Table 1. List of possible factors affecting haemoglobin variability

haemoglobin concentrations outside the target range for more weeks $(13.9 \pm 4.7$ weeks versus $12.5 \pm 5$ weeks, $\mathrm{p}=0.04)$ and (ii) higher standard deviation of haemoglobin $(0.84 \pm 0.35$ versus $0.74 \pm 0.27, p=0.01$ ). Interestingly, in one report, the risk of developing haemoglobin variability was greater with long-acting ESAs (Boudville et al., 2009). De Nicola and colleagues did not find any association between long-acting ESAs versus erythropoietin and haemoglobin variability (De Nicola et al., 2007). However, they observed that baseline haemoglobin level, first dose of ESA and initial iron supplementation were directly associated with the length of time-in-target haemoglobin.

Depending on the 6-group classification based on the highest and lowest categories of haemoglobin, Gilbertson and colleagues reported that patients in the low-intermediate group received high doses of erythropoietin and more blood transfusions (Gilbertson et al., 2009). Minutolo and colleagues found that haemoglobin variability was associated with responsiveness to the first dose of erythropoietin (Minutolo et al., 2009). They also observed that a change of erythropoietin dosage occurred less frequently than expected in spite of regular follow up visits. Therefore, they concluded that lack of adjustment of erythropoietin dosage can lead to haemoglobin variability. However, their data on the effect of frequency and magnitude of adjustment of erythropoietin dosage on haemoglobin stabilisation is less clear. In a post hoc analysis of a randomised controlled trial involving 154 ESKD patients on haemodialysis, more frequent adjustments of erythropoietin dosage as well as larger changes of erythropoietin dosage were associated with haemoglobin variability (Lau et al., 2010).

Using data from the Dialysis Outcomes and Practice Patterns Study (DOPPS) involving 26,510 patients, Pisoni and colleagues studied the associations between facility-level 
anaemia management practices and facility-level haemoglobin standard deviations (Pisoni et. al., 2011). This study identified factors that decreased haemoglobin variability which include reviewing ESA dose at least twice a week and checking haemoglobin levels on a weekly basis. There was also less haemoglobin variability in facilities with a greater percentage of patients prescribed an ESA likely related to better anaemia management with the introduction of an ESA and fewer patients outside the target haemoglobin concentration. The factors that were more likely associated with haemoglobin variability were: facilities with a wider target haemoglobin range, higher upper target haemoglobin, and ESA administration by subcutaneous route (compared to intravenous route).

\subsection{Patient-level factors}

Various studies have reported that young age is a risk factor for haemoglobin variability (De Nicola et al., 2007; Eckardt et al., 2010). Boudville and colleagues reported that the odds of haemoglobin variability decreased by $11 \%$ with each 10 -yr increment in age (Boudville et al., 2009). A few studies have reported that women were more likely to have fluctuations in the haemoglobin concentration (Ebben et al., 2006; Gilbertson et. al., 2008). In a study of 119 non-dialysis CKD patients, male gender was directly associated with increased time-intarget haemoglobin (De Nicola et al., 2007).

Eckardt and colleagues studied the magnitude and frequency of haemoglobin variability as a quantitative index by integrating the area under the curve (AUC) between measured haemoglobin values and the mean haemoglobin concentration (Eckardt et al., 2010). High degree of haemoglobin variability was defined as AUC $>50^{\text {th }}$ percentile. The mean body mass index (BMI) was lowest in the highest quartile of AUC. On multivariate logistic regression, BMI 25 to $30 \mathrm{~kg} / \mathrm{m}^{2}$ and $>30 \mathrm{~kg} / \mathrm{m}^{2}$ were independently associated with decreased odds of haemoglobin variability compared to the reference category of BMI 18 to $25 \mathrm{~kg} / \mathrm{m}^{2}$. Similarly, Lau and colleagues also observed less positive deflection of haemoglobin in heavier patients (Lau et al., 2010).

There is an excess burden of comorbid conditions in CKD, leading to erythropoietin hyporesponsiveness. In an observational study involving 152,846 ESKD patients on haemodialysis, Ebben and colleagues reported that having 2 or more comorbid conditions, 6 or more days of hospitalisation, and occurrence of infectious hospitalisations were independently associated with haemoglobin variability (Ebben et al., 2006).

Eckardt and colleagues found that incident dialysis vintage, change in haemodialysis vascular access, use of catheter for haemodialysis, haemoglobin lower than $11 \mathrm{~g} / \mathrm{dL}$, use of angiotensin-converting enzyme inhibitor or angiotensin receptor blocker, and hospitalisation were positively associated with an increased risk of haemoglobin variability (Eckardt et al., 2010). Also, patients treated with an ESA were twice likely to experience hemoglobin variability than those not treated with an ESA. As expected, higher serum albumin concentration was negatively associated with haemoglobin variability. Interestingly, history of cardiovascular disease was negatively associated with haemoglobin variability. Furthermore, the investigators did not find any association of $\mathrm{C}$-reactive protein and leukocyte count with haemoglobin variability. The reasons for these findings are not entirely clear, but this study included a highly selected cohort in whom complete data on monthly haemoglobin values for 6 mo and medications were available, raising a possibility of selection bias. Similarly, Lau and colleagues also found a positive association between 
catheter use and haemoglobin variability; and a negative association with high baseline haemoglobin (Lau et al., 2010).

Weinhandl and colleagues studied the risk factors for haemoglobin variability in Medicare haemodialysis patients (Weinhandl et. al., 2011). The study cohort included 3 groups of haemodialysis patients: historical prevalent (prevalent on July 1, 1996; $n=78,602$ ), contemporary prevalent (prevalent on July 1, 2006; $n=133,246$ ), and incident (January 1, 2005 - June 30, 2006; $n=24,999)$. In both the prevalent groups, the presence of all comorbid conditions, except hepatic disease, was associated with greater haemoglobin variability. These conditions included atherosclerotic heart disease, congestive heart failure, arrhythmia and other cardiac diseases, cerebrovascular disease, peripheral vascular disease, cancer, chronic obstructive pulmonary disease, diabetes and gastrointestinal bleeding. In the incident group, the presence of cerebrovascular disease, peripheral vascular disease, chronic obstructive pulmonary disease, diabetes and gastrointestinal bleeding were associated with haemoglobin variability. In all 3 groups, cumulative hospital days and number of months with haemoglobin $<10 \mathrm{~g} / \mathrm{dL}$ were positively associated with haemoglobin variability. Similar findings have been reported by Gilbertson and colleagues (Gilbertson et al., 2009).

\subsection{Facility-level factors}

Pisoni and colleagues studied facility-level risk factors for haemoglobin variability in 26,510 haemoglobin patients from 930 facilities in 12 countries using the DOPPS data (Pisoni et al., 2011). Haemoglobin variability was not associated with the number of haemodialysis patients per facility. However, larger differences in mean facility-level haemoglobin standard deviation were seen between countries. The mean age was nearly 2 years younger in the highest quartile of facility-level haemoglobin standard deviation than the lowest quartile. The investigators found that BMI, neutrophil count, and prevalence of psychiatric disorders and hepatitis $C$ were higher in facilities with higher facility-level haemoglobin standard deviation. They also observed a positive association between haemoglobin variability and increased proportion of patients in a facility with parathyroid hormone level $>450 \mathrm{pg} / \mathrm{mL}$. Furthermore, the investigators reported a strong correlation $(r=0.56)$ between facility-level haemoglobin standard deviation and within-patient haemoglobin standard deviation. These findings suggest that the results of this facility-level study could be generalised to an individual patient.

\section{Association between haemoglobin variability and mortality}

An emerging body of evidence suggests that haemoglobin variability is associated with increased risk of all-cause death in both non-dialysis CKD and ESKD patients. Boudville and colleagues found an association between haemoglobin variability and death not only in non-dialysis CKD patients treated with ESAs $(n=1,823)$, but also in those who were not on ESAs $(n=3,143)$ (Boudville et al., 2009). For each $1 \mathrm{~g} / \mathrm{L}$ increase in the residual standard deviation, HR $(95 \% \mathrm{CI})$ for patients on ESA throughout the study and those who were not receiving ESA were 1.02 (1.01 to 1.04) and 1.03 (1.02 to 1.05), respectively. The analysis of the pooled data from these 2 groups showed similar results. Compared to patients with haemoglobin levels consistently within the target range, those with low amplitude fluctuation with low haemoglobin values (HR 1.62, 95\% CI 1.36 to 1.94) and high amplitude fluctuation (HR 1.57, 95\% CI 1.24 to 1.98) were at increased risk of all-cause mortality. 
Minutolo and colleagues reported that longer time with haemoglobin within the target range of 11 to $13 \mathrm{~g} / \mathrm{dL}$ was associated with decreased risk of renal death (defined as a composite endpoint of all-cause death on dialysis or after kidney transplantation) (Minutolo et al., 2009).

In a study involving 34,963 haemodialysis patients who were enrolled in the Fresenius Medical Care database in 1996, Yang and colleagues reported that the risk of all-cause mortality increased proportionately with haemoglobin variability (Yang et al., 2007). The hazard ratio and $95 \%$ confidence intervals $(\mathrm{CI})$ per $0.50 \mathrm{~g} / \mathrm{dL}, 0.75 \mathrm{~g} / \mathrm{dL}, 1.00 \mathrm{~g} / \mathrm{dL}$, and 1.50 $\mathrm{g} / \mathrm{dL}$ increases in haemoglobin variability were 1.15 (1.10 to 1.20$), 1.24$ (1.16 to 1.32 ), 1.33 (1.22 to 1.45), and 1.53 (1.35 to 1.75), respectively.

Gilbertson and colleagues found that out of 6 categories of haemoglobin variability (categorized as low $<11 \mathrm{~g} / \mathrm{dL}$, intermediate $11-12.5 \mathrm{~g} / \mathrm{dL} \&$ high $>12.5 \mathrm{~g} / \mathrm{dL}$ and further divided into low-low, intermediate-intermediate, high-high, low-intermediate, intermediate-high, low-high), patients in the low-high and low-intermediate groups experienced an increased risk of death compared with those in the intermediateintermediate group (Gilbertson et al., 2008). The HR and 95\%CI for the low-high and lowintermediate groups were 1.19 (1.10 to 1.28) and 1.44 (1.33 to 1.56), respectively. Although this categorization broadly identifies patients with stable hemoglobin and either low or high amplitude fluctuations in hemoglobin, it assumes a unidirectional and linear change in hemoglobin.

Lau and colleagues measured haemoglobin variability as rate of haemoglobin change: average positive-only (positive haemoglobin deflections) and average negative-only (negative haemoglobin deflections) (Lau et al., 2010). While negative haemoglobin deflections were not associated with mortality risk (HR 1.07, 95\% CI 0.94 to 1.21 per $\mathrm{g} / \mathrm{L} /$ week), rapid rise in haemoglobin was associated increased risk (HR 1.23, 95\% CI 1.03 to 1.48 per $\mathrm{g} / \mathrm{L} /$ week).

Regidor and colleagues analysed a cohort of 58,058 prevalent haemodialysis patients from the DaVita dialysis organisation (Regidor et al., 2006). Compared to patients whose haemoglobin remain unchanged during the first 6 mo of the 2-year cohort study period, the risks of all-cause and cardiovascular mortality were significantly higher in patients with reduction in haemoglobin by more than $1.50 \mathrm{~g} / \mathrm{dL} /$ quarter. In the fully-adjusted model (adjusted for demographic characteristics, comorbidities, smoking, dialysis dose, nutritional status, iron studies, doses of ESAs and iron), there was no association observed with increment in haemoglobin and mortality. However, decrease in hemoglobin was associated with increased mortality. While this study described an association between change in hemoglobin per quarter and mortality, it did not specifically study an association between hemoglobin variability and mortality.

Pisoni and colleagues reported mortality outcomes using haemoglobin variability at the facility-level using the DOPPS database (Pisoni et al., 2011). In the adjusted model, the HR for every $0.5 \mathrm{~g} / \mathrm{dL}$ higher facility-level haemoglobin standard deviation was 1.08 (95\% CI 1.02 to 1.15). Compared to the reference category of the lowest quartile of facility-level haemoglobin standard deviation, the $\mathrm{HR}(95 \% \mathrm{CI})$ for the $2^{\text {nd }}, 3^{\text {rd }}$ and $4^{\text {th }}$ quartiles were 1.08 (1.10 to 1.34 ), 1.15 (1.35 to 1.69 ) and 1.19 (1.04 to 1.37), respectively. As previously mentioned, the facility-level haemoglobin standard deviation correlated well with withinpatient haemoglobin standard deviation. 
Not all studies have demonstrated a positive association between haemoglobin variability and death in CKD. Eckardt and colleagues studied the effect of haemoglobin variability on mortality using 5 definitions: within-patient standard deviation, residual standard deviation, time-in-target haemoglobin, amplitudes of fluctuation, and AUC (Eckardt et al., 2010). In the adjusted Cox regression model, haemoglobin variability was not a statistically significant factor in all 5 methods, except for the group of patients with low amplitude fluctuations with low haemoglobin levels (HR 1.74, 95\% CI 1.00 to 3.04). However, 95\% confidence intervals were very wide and the lower 95\% confidence interval was 1.00. Thus, although this association was statistically significant, it was weak.

Brunelli and colleagues included a retrospective cohort of 6,644 incident patients who commenced haemodialysis between 2004 and 2005 from the Fresenius Medical Centre database (Brunelli et. al., 2008). In contrast to their 1996 cohort study results (Yang et al., 2007), the association between haemoglobin variability and mortality was not statistically significant. The unadjusted and adjusted HR $(95 \% \mathrm{CI})$ for were $0.96(0.81$ to 1.14$)$ and 1.11 (0.92 to 1.33), respectively. The discrepancy in these analyses may be explained by the addition of a large number of variables in the Cox regression model for the 2004-2005 cohort as more data were available. Consequently, when the analysis was restricted using the same limited variables as in the 1996 cohort study; the association achieved statistical significance with a HR of 1.22 (1.01 to 1.48). Although the investigators have attempted to adjust for known variables, the possibility of residual confounding could not be excluded. The complexity of these statistical models makes interpretation of the results difficult, particularly when the different statistical methods or approaches did not generate robust or consistent findings.

Weinhandl and colleagues reported the association between haemoglobin variability and all-cause mortality using 3 Cox proportional hazards regression models (Weinhandl et al., 2011). In the case-mix-adjusted model, the HR (95\% CI) for the contemporary prevalent, historical prevalent and incident groups for $1 \mathrm{~g} / \mathrm{dL}$ haemoglobin variability were 1.27 (1.24 to 1.31$), 1.32$ (1.27 to 1.38 ) and 1.08 (1.03 to 1.13), respectively. In the comorbid conditionadjusted model, haemoglobin variability was associated with increased risk of death in both the prevalent groups with a HR of 1.07 (1.04 to 1.10) in the contemporary prevalent group and 1.10 (1.06 to 1.15) in the historical prevalent group. However, there was no statistically significant association in the incident group (HR 1.03, 95\% CI 0.98 to 1.09). In the expanded comorbid condition-adjusted model, the statistically significant association was limited only to the historical prevalent group (HR 1.07, 95\% CI 1.03 to 1.12). Haemoglobin variability was not associated with increased risk of death in either the contemporary prevalent (HR 1.02, $95 \% \mathrm{CI} 0.99$ to 1.05 ) or incident groups (HR 1.01, 95\% CI 0.95 to 1.06). These findings suggest that the association between haemoglobin variability and mortality was weak in this study and was sensitive to adjustment for concurrent disease severity.

\section{Management of haemoglobin variability}

Since the introduction of ESAs, most of the clinical trials with ESA therapy have focused on haemoglobin targets in CKD patients. There is a shortage of clinical trials studying the optimal strategy for haemoglobin monitoring in patients treated with ESAs and interventions to reduce haemoglobin variability. 
Ho and colleagues conducted an observational case-control study of unselected ESKD patients on haemodialysis (Ho et. al., 2010). Patients served as their own control. They compared $2 x /$ month laboratory haemoglobin measurements and use of a computerised algorithm to analyse $12 x$ /month monitoring of Crit-line haemoglobin measurements. They found that haemoglobin variability, measured by the mean standard deviation of the residuals, significantly improved during the phase of frequent haemoglobin monitoring. However, this was not a randomised controlled trial. Also, the sample size was small and it was an unblinded study in a single-centre. Nonetheless, the study highlights the importance of frequent monitoring of haemoglobin as it may provide an opportunity to titrate the ESA dose early.

Gaweda and colleagues conducted a case-controlled observational study in which 49 ESKD patients on haemodialysis were included (Gaweda et. al., 2010). The investigators measured haemoglobin using Crit-line at various intervals: twice weekly or $8 \mathrm{x} / \mathrm{mo}$, once weekly or $4 x /$ mo, every 2 weeks or $2 x /$ mo and every 4 weeks or $1 x /$ mo. They also calculated the haemoglobin estimation error as a root mean-squared difference between the observed and estimated haemoglobin and compared it with the measurement error. They found that the most accurate haemoglobin estimation was achieved with twice weekly haemoglobin sampling, although it exceeded the accuracy of the measurement device. Twice and once weekly haemoglobin measurements were found to be optimal in $31 \%$ and $45 \%$ patients, respectively. This was also a single-centre study with a small sample size.

These two studies highlight the paucity of evidence in the area of management of haemoglobin variability. Considering the cost and possible amount of blood loss associated with frequent hemoglobin monitoring, currently available evidence is not sufficient to make any clinical practice recommendations.

\section{Future research}

There is substantial heterogeneity in the haematological response to ESAs. Although the factors underpinning ESA-hyporesponsiveness are well characterised, those contributing to haemoglobin variability are poorly understood. Future studies should include prospective and systematic data collection to evaluate these factors. Investigations should also be carried out to develop a single and uniformly accepted method to measure haemoglobin variability that is clinically relevant and reproducible, since there is no consensus on a single method for the measurement of haemoglobin variability.

The data on the effect of haemoglobin variability on mortality are conflicting. This could be due to several reasons. Firstly, most of the data originates from retrospective and observational studies. Secondly, the studies are arbitrarily limited to sampling during 6 month periods where monthly haemoglobin values for each month were available. This cross-sectional nature of the study does not reflect long-term fluctuations in haemoglobin values. Thirdly, due to highly selective nature of the study cohorts, sampling bias could be potentially introduced. Most of these studies had a very short follow up period ranging from 6 months to 18 months. Furthermore, different statistical models have demonstrated inconsistent and non-reproducible results due considerable between-study variation in the covariates included in the adjusted models. Therefore, the exact nature and quantification of the effect of haemoglobin variability on mortality is still poorly understood. 
Future studies should be designed carefully and conducted prospectively to define the exact magnitude of the effect of haemoglobin variability on mortality. They should also include a greater breadth and depth of the CKD patient populations since dialysis studies have not included patients receiving peritoneal dialysis and studies involving non-dialysis CKD patients have been very limited.

Most of the current data is merely hypothesis generating. Therefore, well designed and adequately powered randomised controlled trials are needed to determine the optimal frequency of haemoglobin monitoring along with a cost-effective analysis. Further trials are required to define the optimal strategy of frequency and magnitude of ESA and iron dose changes, and study their possible interactions with concurrent acute illness and the presence of comorbid conditions.

\section{Summary}

Haemoglobin variability is highly prevalent in both non-dialysis and dialysis CKD patients. The factors contributing to haemoglobin variability are not entirely clear. However, the currently available evidence has identified the following factors: younger age, female gender, low body mass index, the presence of comorbid conditions, and the use of ESA and less frequent monitoring of haemoglobin. There is conflicting evidence on the effect of haemoglobin variability on mortality with some studies demonstrating a strong association and others showing no association with mortality. A few small prospective observational studies have found that frequent monitoring of haemoglobin may reduce short-term haemoglobin variability, although the optimal frequency of haemoglobin monitoring is yet to be defined. Evidence-based treatment strategies cannot be currently recommended due to a lack of high quality data. In conclusion, maintaining haemoglobin within a target range continues to pose a challenge to clinicians. Further research is urgently needed in this insufficiently researched field.

\section{Acknowledgment}

Professor David Johnson is a recipient of consultancy fees, research grants, travel sponsorships and speakers' honoraria from Amgen, Roche and Janssen-Cilag. He is a recipient of consultancy fees from Sandoz and is a co-investigator on industry-sponsored research by Amgen, Roche and Janssen-Cilag. He is currently supported by a Queensland Government Health Research Fellowship. Other authors do not have any conflicts of interest to declare.

\section{References}

Badve, S. V., Hawley, C. M. , \& Johnson, D. W. (2011). Is the problem with the vehicle or the destination? Does high-dose ESA or high haemoglobin contribute to poor outcomes in CKD? Nephrology (Carlton), Vol.16, No.2, pp. 144-153

Besarab, A., Bolton, W. K., Browne, J. K., Egrie, J. C., Nissenson, A. R., Okamoto, D. M., Schwab, S. J. , \& Goodkin, D. A. (1998). The effects of normal as compared with low 
hematocrit values in patients with cardiac disease who are receiving hemodialysis and epoetin. N Engl J Med, Vol.339, No.9, pp. 584-590

Boudville, N. C., Djurdjev, O., Macdougall, I. C., de Francisco, A. L., Deray, G., Besarab, A., Stevens, P. E., Walker, R. G., Urena, P., Inigo, P., Minutolo, R., Haviv, Y. S., Yeates, K., Aguera, M. L., MacRae, J. M. , \& Levin, A. (2009). Hemoglobin variability in nondialysis chronic kidney disease: examining the association with mortality. Clin J Am Soc Nephrol, Vol.4, No.7, pp. 1176-1182

Brunelli, S. M., Lynch, K. E., Ankers, E. D., Joffe, M. M., Yang, W., Thadhani, R. I. , \& Feldman, H. I. (2008). Association of hemoglobin variability and mortality among contemporary incident hemodialysis patients. Clin J Am Soc Nephrol, Vol.3, No.6, pp. 1733-1740

Clement, F. M., Klarenbach, S., Tonelli, M., Johnson, J. A. , \& Manns, B. J. (2009). The impact of selecting a high hemoglobin target level on health-related quality of life for patients with chronic kidney disease: a systematic review and meta-analysis. Arch Intern Med, Vol.169, No.12, pp. 1104-1112

De Nicola, L., Conte, G., Chiodini, P., Cianciaruso, B., Pota, A., Bellizzi, V., Tirino, G., Avino, D., Catapano, F. , \& Minutolo, R. (2007). Stability of target hemoglobin levels during the first year of epoetin treatment in patients with chronic kidney disease. Clin J Am Soc Nephrol, Vol.2, No.5, pp. 938-946

Ebben, J. P., Gilbertson, D. T., Foley, R. N. , \& Collins, A. J. (2006). Hemoglobin level variability: associations with comorbidity, intercurrent events, and hospitalizations. Clin J Am Soc Nephrol, Vol.1, No.6, pp. 1205-1210

Eckardt, K. U., Kim, J., Kronenberg, F., Aljama, P., Anker, S. D., Canaud, B., Molemans, B., Stenvinkel, P., Schernthaner, G., Ireland, E., Fouqueray, B. , \& Macdougall, I. C. (2010). Hemoglobin variability does not predict mortality in European hemodialysis patients. J Am Soc Nephrol, Vol.21, No.10, pp. 1765-1775

Eschbach, J. W., Egrie, J. C., Downing, M. R., Browne, J. K. , \& Adamson, J. W. (1987). Correction of the anemia of end-stage renal disease with recombinant human erythropoietin. Results of a combined phase I and II clinical trial. $N$ Engl J Med, Vol.316, No.2, pp. 73-78

Gandra, S. R., Finkelstein, F. O., Bennett, A. V., Lewis, E. F., Brazg, T. , \& Martin, M. L. (2010). Impact of erythropoiesis-stimulating agents on energy and physical function in nondialysis CKD patients with anemia: a systematic review. Am J Kidney Dis, Vol.55, No.3, pp. 519-534

Gaweda, A. E., Nathanson, B. H., Jacobs, A. A., Aronoff, G. R., Germain, M. J. , \& Brier, M. E. (2010). Determining optimum hemoglobin sampling for anemia management from every-treatment data. Clin J Am Soc Nephrol, Vol.5, No.11, pp. 1939-1945

Gilbertson, D. T., Peng, Y., Bradbury, B., Ebben, J. P. , \& Collins, A. J. (2009). Hemoglobin level variability: anemia management among variability groups. Am J Nephrol, Vol.30, No.6, pp. 491-498

Gilbertson, D. T., Ebben, J. P., Foley, R. N., Weinhandl, E. D., Bradbury, B. D. , \& Collins, A. J. (2008). Hemoglobin level variability: associations with mortality. Clin J Am Soc Nephrol, Vol.3, No.1, pp. 133-138

Ho, W. R., Germain, M. J., Garb, J., Picard, S., Mackie, M. K., Bartlett, C. , \& Will, E. J. (2010). Use of $12 x /$ month haemoglobin monitoring with a computer algorithm 
reduces haemoglobin variability. Nephrol Dial Transplant, Vol.25, No.8, pp. 27102714

Kalantar-Zadeh, K. \& Aronoff, G. R. (2009). Hemoglobin variability in anemia of chronic kidney disease. J Am Soc Nephrol, Vol.20, No.3, pp. 479-487

Lacson, E., Jr., Ofsthun, N. , \& Lazarus, J. M. (2003). Effect of variability in anemia management on hemoglobin outcomes in ESRD. Am J Kidney Dis, Vol.41, No.1, pp. 111-124

Lau, J. H., Gangji, A. S., Rabbat, C. G. , \& Brimble, K. S. (2010). Impact of haemoglobin and erythropoietin dose changes on mortality: a secondary analysis of results from a randomized anaemia management trial. Nephrol Dial Transplant, Vol.25, No.12, pp. 4002-4009

Minutolo, R., Chiodini, P., Cianciaruso, B., Pota, A., Bellizzi, V., Avino, D., Mascia, S., Laurino, S., Bertino, V., Conte, G. , \& De Nicola, L. (2009). Epoetin therapy and hemoglobin level variability in nondialysis patients with chronic kidney disease. Clin J Am Soc Nephrol, Vol.4, No.3, pp. 552-559

Palmer, S. C., Navaneethan, S. D., Craig, J. C., Johnson, D. W., Tonelli, M., Garg, A. X., Pellegrini, F., Ravani, P., Jardine, M., Perkovic, V., Graziano, G., McGee, R., Nicolucci, A., Tognoni, G. , \& Strippoli, G. F. (2010). Meta-analysis: erythropoiesisstimulating agents in patients with chronic kidney disease. Ann Intern Med, Vol.153, No.1, pp. 23-33

Patel, T., Hirter, A., Kaufman, J., Keithi-Reddy, S. R., Reda, D. , \& Singh, A. (2009). Route of epoetin administration influences hemoglobin variability in hemodialysis patients. Am J Nephrol, Vol.29, No.6, pp. 532-537

Pfeffer, M. A., Burdmann, E. A., Chen, C. Y., Cooper, M. E., de Zeeuw, D., Eckardt, K. U., Feyzi, J. M., Ivanovich, P., Kewalramani, R., Levey, A. S., Lewis, E. F., McGill, J. B., McMurray, J. J., Parfrey, P., Parving, H. H., Remuzzi, G., Singh, A. K., Solomon, S. D. , \& Toto, R. (2009). A trial of darbepoetin alfa in type 2 diabetes and chronic kidney disease. N Engl J Med, Vol.361, No.21, pp. 2019-2032

Phrommintikul, A., Haas, S. J., Elsik, M. , \& Krum, H. (2007). Mortality and target haemoglobin concentrations in anaemic patients with chronic kidney disease treated with erythropoietin: a meta-analysis. Lancet, Vol.369, No.9559, pp. 381-388

Pisoni, R. L., Bragg-Gresham, J. L., Fuller, D. S., Morgenstern, H., Canaud, B., Locatelli, F., Li, Y., Gillespie, B., Wolfe, R. A., Port, F. K. , \& Robinson, B. M. (2011). Facilitylevel interpatient hemoglobin variability in hemodialysis centers participating in the Dialysis Outcomes and Practice Patterns Study (DOPPS): Associations with mortality, patient characteristics, and facility practices. Am J Kidney Dis, Vol.57, No.2, pp. 266-275

Regidor, D. L., Kopple, J. D., Kovesdy, C. P., Kilpatrick, R. D., McAllister, C. J., Aronovitz, J., Greenland, S. , \& Kalantar-Zadeh, K. (2006). Associations between changes in hemoglobin and administered erythropoiesis-stimulating agent and survival in hemodialysis patients. J Am Soc Nephrol, Vol.17, No.4, pp. 1181-1191

Singh, A. K., Szczech, L., Tang, K. L., Barnhart, H., Sapp, S., Wolfson, M. , \& Reddan, D. (2006). Correction of anemia with epoetin alfa in chronic kidney disease. $N$ Engl J Med, Vol.355, No.20, pp. 2085-2098 
Weinhandl, E. D., Peng, Y., Gilbertson, D. T., Bradbury, B. D. , \& Collins, A. J. (2011). Hemoglobin variability and mortality: confounding by disease severity. Am J Kidney Dis, Vol.57, No.2, pp. 255-265

Yang, W., Israni, R. K., Brunelli, S. M., Joffe, M. M., Fishbane, S. , \& Feldman, H. I. (2007). Hemoglobin variability and mortality in ESRD. J Am Soc Nephrol, Vol.18, No.12, pp. 3164-3170 


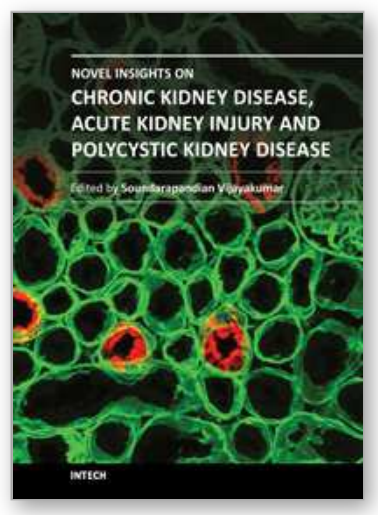

\section{Novel Insights on Chronic Kidney Disease, Acute Kidney Injury and Polycystic Kidney Disease}

Edited by Dr. Soundarapandian Vijayakumar

ISBN 978-953-51-0234-2

Hard cover, 134 pages

Publisher InTech

Published online 07, March, 2012

Published in print edition March, 2012

This book offers novel insights on topics such as congenital obstructive nephropathy, cerebral-renal salt wasting, and the role of hemoglobin variability in clinical outcomes of CKD which are not very often discussed in the literature. With comprehensive and insightful reviews by eminent clinicians and scientists in the field, this book is a valuable tool for nephrologists.

\section{How to reference}

In order to correctly reference this scholarly work, feel free to copy and paste the following:

Roshini Malasingam, David W. Johnson and Sunil V. Badve (2012). Association Between Haemoglobin Variability and Clinical Outcomes in Chronic Kidney Disease, Novel Insights on Chronic Kidney Disease, Acute Kidney Injury and Polycystic Kidney Disease, Dr. Soundarapandian Vijayakumar (Ed.), ISBN: 978-953-510234-2, InTech, Available from: http://www.intechopen.com/books/novel-insights-on-chronic-kidney-diseaseacute-kidney-injury-and-polycystic-kidney-disease/association-between-hemoglobin-variability-and-clinicaloutcomes-in-chronic-kidney-disease

\section{INTECH}

open science | open minds

\section{InTech Europe}

University Campus STeP Ri

Slavka Krautzeka 83/A

51000 Rijeka, Croatia

Phone: +385 (51) 770447

Fax: +385 (51) 686166

www.intechopen.com

\section{InTech China}

Unit 405, Office Block, Hotel Equatorial Shanghai

No.65, Yan An Road (West), Shanghai, 200040, China

中国上海市延安西路 65 号上海国际贵都大饭店办公楼 405 单元

Phone: +86-21-62489820

Fax: +86-21-62489821 
(C) 2012 The Author(s). Licensee IntechOpen. This is an open access article distributed under the terms of the Creative Commons Attribution 3.0 License, which permits unrestricted use, distribution, and reproduction in any medium, provided the original work is properly cited. 